\title{
Interface thermal resistance between dissimilar anharmonic lattices
}

\author{
Baowen $\operatorname{Li}^{1,2,4} *$ Jinghua $\operatorname{Lan}^{1}$, and Lei Wang ${ }^{3,4}$ \\ 1 Department of Physics, National University of Singapore, Singapore 117542, Republic of Singapore \\ ${ }^{2}$ NUS Graduate School for Integrative Sciences and Engineering, Singapore 117597, Republic of Singapore \\ 3 Temasek Laboratories, National University of Singapore, Singapore 119260, Republic of Singapore \\ 4 The Beijing-Hong Kong-Singapore Joint Center for Nonlinear and Complex Systems (Singapore), Singapore 117542
}

(Dated: Published in Phys. Rev. Lett, 95, 104302 (2005))

\begin{abstract}
We study interface thermal resistance (ITR) in a system consisting of two dissimilar anharmonic lattices exemplified by Fermi-Pasta-Ulam (FPU) model and Frenkel-Kontorova (FK) model. It is found that the ITR is asymmetric, namely, it depends on how the temperature gradient is applied. The dependence of the ITR on the coupling constant, temperature, temperature difference, and system size are studied. Possible applications in nanoscale heat management and control are discussed.
\end{abstract}

PACS numbers: 67.40.Pm, 63.20.Ry, 66.70.+f, 44.10.+i,

When heat flows across an interface between two different materials (phases), there exists a temperature jump at the interface, from which we can define an interface thermal resistance (ITR):

$$
R \equiv \frac{\Delta T}{J}
$$

where $J$ is the heat flux density, namely, the heat flow across a unit area in unit time, $\Delta T$ is the temperature difference between two sides of the interface. This problem has been caught attention as early as 1941 when Kapitza [1] discovered the temperature jump at an interface between solid and liquid. Continuous efforts have been devoted to this problem (see Review article 2], and the references therein) since then. More recently, the temperature jump between liquid and vapor has also been observed experimentally $[3]$ and studied by computer simulations [4].

A general theory to describe the temperature jump at interface of different materials (in different phases) is still lacking. There are two popular theories for harmonic lattice: diffuse mismatch theory 2 2 and acoustic mismatch theory [5]. The acoustic mismatch theory regards the two media as two elastic continua, while the diffuse mismatch theory assumes that at the interface all phonons are diffusively scattered. When anharmonic interaction is taken into account, superposition theorem fails, thus no analytic theory can be worked out. However, as we shall show in the following that, if the anharmonic interaction is considered, more interesting phenomena arise, the problem becomes theoretically more challenging, and of course it is closer to reality because harmonic is just a first order approximation. On the other hand, as the rapid development of nanotechnology, low dimensional nano scale systems such as nanowires and nanotubes can be easily fabricated in the lab. At the nano scale, the systems are of finite size, more precisely, they are discrete, and therefore, the continuous theory such as the acoustic mismatch theory will definitely not be suitable for such systems. The interface resistance in such nano systems becomes more and more important and has potential applications in nanoscale heat control and management [6].

In this Letter, we study the ITR in the system comprising two dissimilar anharmonic lattices. The system, illustrated in Fig. 1, consists of two chains of $N$ oscillators. The left part is a chain of $N$ harmonic oscillators on a substrate whose interaction is represented by a sinusoidal on-site potential. The right part is a chain of $N$ anharmonic oscillators. The two parts are connected by a spring of constant $k_{i n t}$, and the Hamiltonian of the system is,

$$
H=H_{F K}+H_{F P U}+\frac{1}{2} k_{i n t}\left(x_{N+1}-x_{N}-a\right)^{2},
$$

where $H_{F K}$ is the Hamiltonian of the left part which is in fact the Frenkel Kontorova (FK) model, $H_{F K}=\sum_{i=1}^{N} \frac{p_{i}^{2}}{2 m}+\frac{1}{2} k_{F K}\left(x_{i}-x_{i-1}-b\right)^{2}-\frac{V}{(2 \pi)^{2}} \cos 2 \pi x_{i}$. $H_{F P U}$ is the Hamiltonian of the right part which is the Fermi-Pasta-Ulam (FPU) model, $H_{F P U}=\sum_{i=N+1}^{2 N} \frac{p_{i}^{2}}{2 m}+$ $k_{F P U}\left[\frac{1}{2}\left(x_{i+1}-x_{i}-a\right)^{2}+\frac{\beta}{4}\left(x_{i+1}-x_{i}-a\right)^{4}\right]$.

Fixed boundaries are used, i.e. $x_{0}=0$ and $x_{2 N+1}=N b+(N+1) a$. The FPU model is a representative anharmonic lattice without on-site potential and the FK model is the one with on-site potential. Both models have been widely used to study different problems in condensed matter physics and nonlinear dynamics [7, 8]. In particular, the FPU model has played an important role in the development of computational physics and nonlinear dynamics 7]. Recent years have witnessed an increasing interests in the study of heat conduction with these two models $9,10,11,12,13]$.

In our simulation, Nosé-Hoover heat baths are used. The system parameters are $k_{F K}=1, \beta=1, a=b=1$, $m=1$, and $V=5 . T_{L}=T_{0}(1+\Delta), T_{R}=T_{0}(1-\Delta)$. $k_{F P U}=0.2$ unless otherwise stated. The local temperature at site $n$ is defined as $T_{n}=m\left\langle\dot{x_{n}}{ }^{2}\right\rangle$, where \langle\rangle stands for temporal average. We calculate the physical quantities after a time that is long enough to allow the system to reach a non-equilibrium steady state where the local 


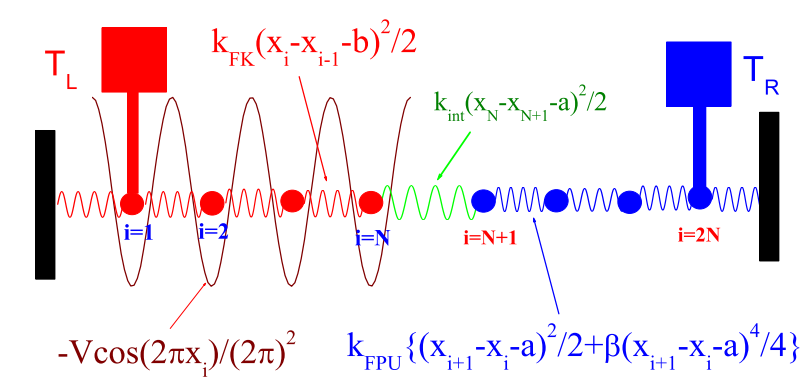

FIG. 1: Configuration of the system. The left part is a Frenkel-Kontorova model and the right one is a Fermi-PastaUlam model. The two parts are connected by a spring of constant $k_{\text {int }}$. The two particles on the left and right ends are put into contact with heat baths of temperature $T_{L}$ and $T_{R}$, respectively.

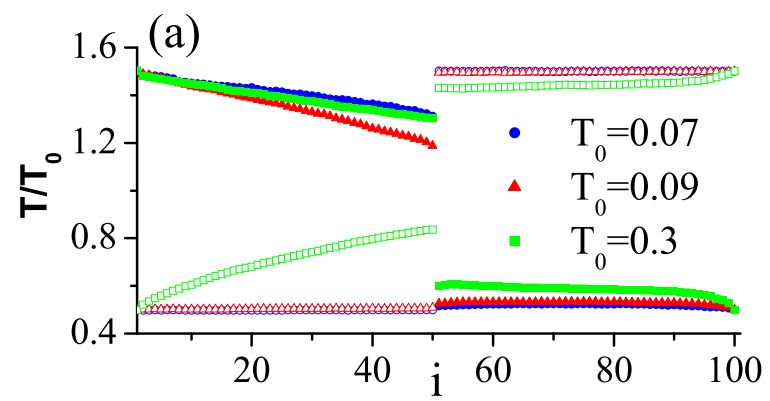

(b)

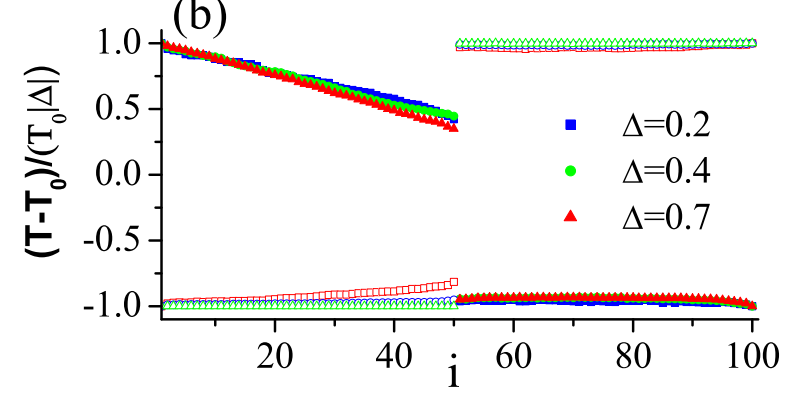

FIG. 2: (a) $T / T_{0}$ versus lattice site for $T_{0}=0.07,0.09$, and 0.3. $|\Delta|=0.5$. (b) $\left(T-T_{0}\right) /\left(T_{0}|\Delta|\right)$ versus lattice site, for different $|\Delta|=0.2,0.4$, and 0.7 with fixed $T_{0}=0.09$. The solid symbols are for the cases of $\Delta>0$, and the empty ones are for the cases of $\Delta<0$. In both (a) and (b) $N=50$.

heat flux is constant along the chain.

Fig[2(a) shows the normalized temperature, $T / T_{0}$, along the lattice site for three different temperatures, $T_{0}=0.07,0.09$, and 0.3 , with fixed $|\Delta|=0.5$. Fig. 2(b) shows $\left(T-T_{0}\right) /\left(T_{0}|\Delta|\right)$ versus lattice site for $|\Delta|=0.2$, 0.4 , and 0.7 with fixed $T_{0}=0.09$. It is obvious that, in all cases, there exists a temperature jump (discontinuity) at the interface. The jump depends not only on temperature $T_{0}$ but also the temperature difference $\Delta$. The most interesting thing is that, the jump is asymmetric, namely, it depends on whether $\Delta$ is positive or negative.
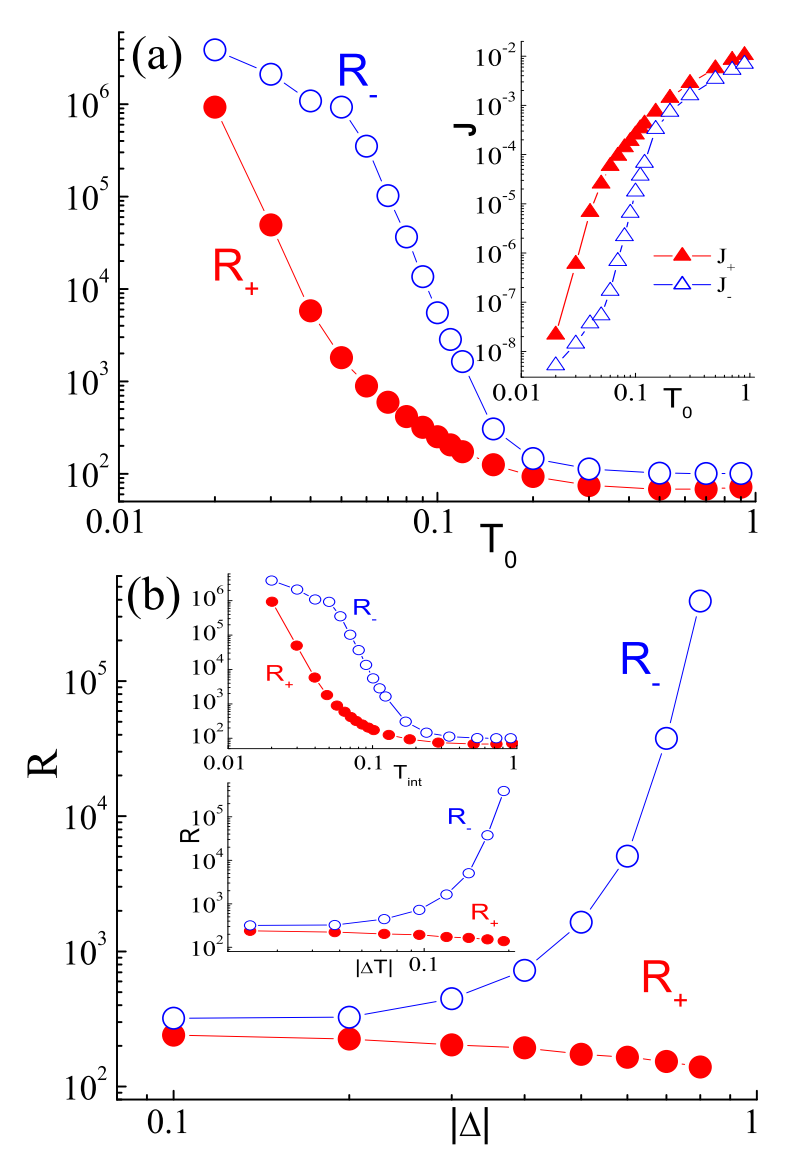

FIG. 3: (a) $R_{ \pm}$versus $T_{0} .|\Delta|=0.5$. Inset is heat current $J_{ \pm}$ versus $T_{0}$. (b) $R_{ \pm}$versus $|\Delta|$ for $T_{0}=0.12$. Inset of (b) is $R_{ \pm}$ versus interface temperature $T_{i n t}=\left(T_{i n t}^{L}+T_{i n t}^{R}\right) / 2$, and $R_{ \pm}$ versus interface temperature jump, $\Delta T$. In all cases $N=50$.

The asymmetric feature is well captured by ITR shown in Fig. $3 R_{ \pm}$denotes the ITR when $\Delta>0$ and $\Delta<0$, respectively. Fig 3 (a) shows that, at intermediate temperature, $T_{0} \in(0.02,0.1)$, the ratio, $R_{-} / R_{+}$, changes from about 10 to 1000 . Both $R_{+}$and $R_{-}$decrease with $T_{0}$ until $T_{0} \approx 0.2$ and then become approximately constants. The dependence of $R_{ \pm}$can be explained from the temperature dependence of the phonon spectra, as we shall discuss fully later on. In the inset of Fig $3(a)$, we show the heat currents $J_{ \pm}$versus temperature $T_{0} . J_{ \pm}$is the heat flux for $\Delta>0$ and $\Delta<0$, respectively. Heat currents increase very fast (in several orders of magnitude) as $T_{0}$ is increased over two orders of magnitude.

In Fig. 3(b), we draw $R_{ \pm}$versus $|\Delta|$ for $T_{0}=0.12$. It shows that as $|\Delta|$ increases, $R_{+}$does not change too much, it is always in the order of 200 , whereas $R_{-}$increases more than three orders of magnitude. In the case of $|\Delta|=0.8, R_{-} / R_{+}$can be larger than 1,000 and $\left|J_{+}\right| \approx 2,000\left|J_{-}\right|$. The dependence of ITR on the interface temperature, $T_{i n t}$ and the interface temperature jump, $\Delta T \equiv T_{i n t}^{L}-T_{i n t}^{R}$ are shown in the inset of Fig. 3(b). Where $T_{\text {int }}^{L, R}$ is the temperature for the particle on 
the left/right side of the interface, respectively.

An obvious conclusion from above results is that the ITR between dissimilar anharmonic lattices is asymmetric.

To understand the physical mechanism of this phenomenon, we need to invoke the energy band theory. However, due to the presence of anharmonicity, an analytic approach seems impossible. We shall rather take a qualitative approach, which can also give us useful information in two extreme cases, namely, low temperature limit (regime) and high temperature limit (regime).

FK model. At very low temperature, the particles are confined in the valleys of the on-site potential. By linearizing equations of motion one can easily obtain the phonon band, $\sqrt{V}<\omega_{F K}^{L}<\sqrt{V+4 k_{F K}}$. On the other hand, in the high temperature limit, the particles have large enough kinetic energies to jump out the valleys. The on-site potential becomes negligible, the FK model degenerates to an harmonic one, $0<\omega_{F K}^{H}<2 \sqrt{k_{F K}}$.

FPU model. There exists a threshold temperature $T_{t h}(\approx 0.1)[10$, below which the FPU model becomes a harmonic one, one thus has $0<\omega_{F P U}^{L}<2 \sqrt{k_{F P U}}$. When $T_{0}>T_{t h}$, the anharmonic term is dominant. In this regime, a rough theoretical estimate yields $0<\omega_{F P U}^{H}<$ $C_{0}\left(T k_{F P U} \beta\right)^{1 / 4}$ with $C_{0}=2 \sqrt{2 \pi} \Gamma(3 / 4) 3^{1 / 4} / \Gamma(1 / 4) \approx$ 2.23 , where $\Gamma$ is the Gamma function.

The spectra of the interface particles are shown in Fig [1(a) and (b) for $\Delta>0$ and $\Delta<0$, respectively, and compared with the above analytical analysis (the shadow regions). In the first case, when $T_{L}=0.15$ and $T_{R}=0.01$, we can approximately regard the FK lattice as at high temperature limit, and the FPU lattice at low temperature regime. With $k_{F K}=1$ and $k_{F P U}=0.2$, we have, $\omega_{F K}^{H} \in[0,2]$, and $\omega_{F P U}^{L} \in[0,0.89]$, which are quite close to the numerical ones.

On the other hand, when the temperatures of the two thermal baths are swapped. Approximately, the FK lattice is at low temperature limit, and the FPU is at high temperature regime. In this case, according to above analysis, the phonon band of the FK model is $\omega_{F K}^{L} \in[2.24,3.00]$ for $V=5$ and $k_{F K}=1$, and $\omega_{F P U}^{H} \in[0,0.94]$ with $T \approx 0.15$ and $\beta=1$ for the FPU model. They are also very close to the numerical results shown in Fig 4 (b).

It is clear from Fig 4 that, in the case of $T_{L}>T_{R}$, the phonon band of the FK lattice overlaps that one of the FPU lattice, therefore, heat can easily flow from the FK lattice to the FPU lattice as is demonstrated in Fig 3 by small $R_{+}$and large $J_{+}$. Conversely, when $T_{L}<T_{R}$, by appropriately chosen parameters, a large gap between the phonon bands of the FK lattice and the FPU lattice can be formed (see Fig 4(b)), which inhibits heat flow from the FPU lattice to the FK lattice as is manifested by a large $R_{-}$and small $J_{-}$in Fig 3 However, as $T_{0}$ increases, the gap becomes narrower and narrower, and eventually disappears when $T_{0}$ surpasses a certain value.
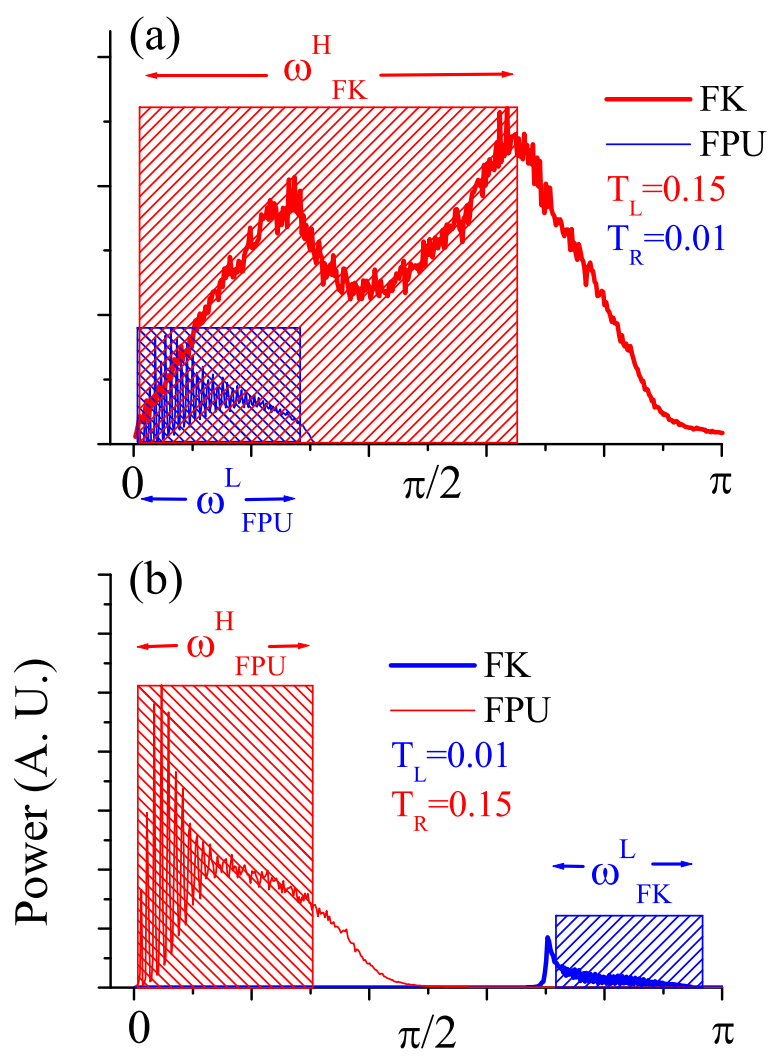

FIG. 4: Phonon spectra of the two particles at interface and schematic phonon bands for the FK and the FPU models in two extremes. (a) $T_{L}=0.15$ and $T_{R}=0.01$. (b) $T_{L}=$ 0.01 and $T_{R}=0.15$. The shadow regions are the analytical estimates for the FK and FPU models. (see text for more explanation.)

Indeed, when $T_{0}$ is large enough the two phonon bands overlap leading to a constant $R_{-}$as is seen in Fig[3(a).

In order to quantify above asymmetric ITR, heat currents, and find the relationships with the overlap of the phonon bands of the two lattices, we introduce,

$$
S_{ \pm}=\frac{\int_{0}^{\infty} P_{l}(\omega) P_{r}(\omega) d \omega}{\int_{0}^{\infty} P_{l}(\omega) d \omega \int_{0}^{\infty} P_{r}(\omega) d \omega} .
$$

$S_{ \pm}$correspondes to the overlap for $\Delta>0$ and $\Delta<0$, respectively. Note that $\int_{0}^{\infty} P_{l, r} d \omega=T_{i n t}^{L, R}$. In Fig 5 we plot $R_{-} / R_{+}$versus $S_{+} / S_{-}$and in the inset we show $\left|J_{+} / J_{-}\right|$ versus $S_{+} / S_{-}$. Best fit gives $R_{-} / R_{+} \sim\left(S_{+} / S_{-}\right)^{\delta_{R}}$ with $\delta_{R}=1.68 \pm 0.08$, and $\left|J_{+} / J_{-}\right| \sim\left(S_{+} / S_{-}\right)^{\delta_{J}}$, with $\delta_{J}=1.62 \pm 0.10$. This picture indeed illustrates that the ITR and heat current correlate strongly with the overlap of the phonon spectra of the two interface particles.

As the system contains many adjustable parameters, it is worth investigating the dependence of the ITR on these parameters. Fig. 6(a) is $R_{ \pm}$versus $k_{i n t}$, which shows both $R_{ \pm}$decreases with increasing $k_{i n t}$. This means that strong coupling favorites heat transport. Fig 6 (b) shows 


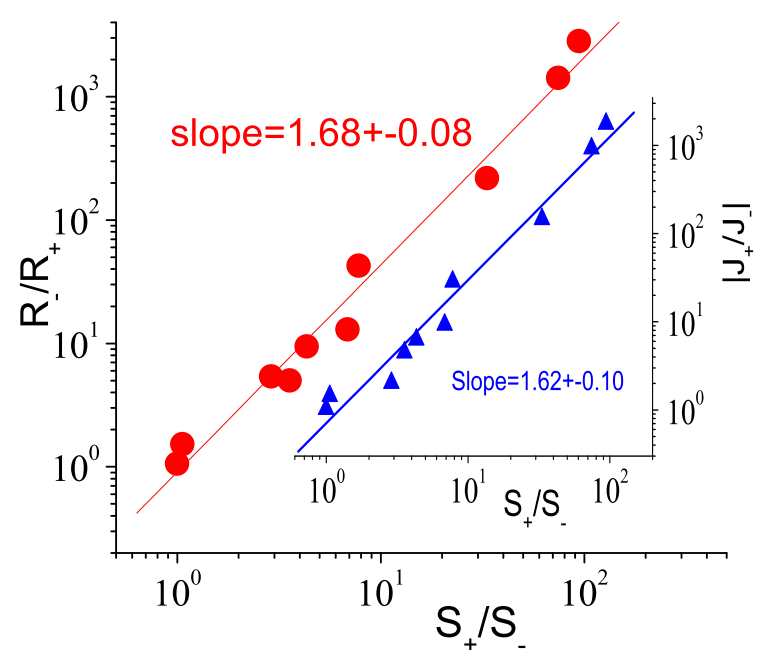

FIG. 5: $R_{-} / R_{+}$versus $S_{+} / S_{-}$. Inset, $\left|J_{+} / J_{-}\right|$versus $S_{+} / S_{-}$.

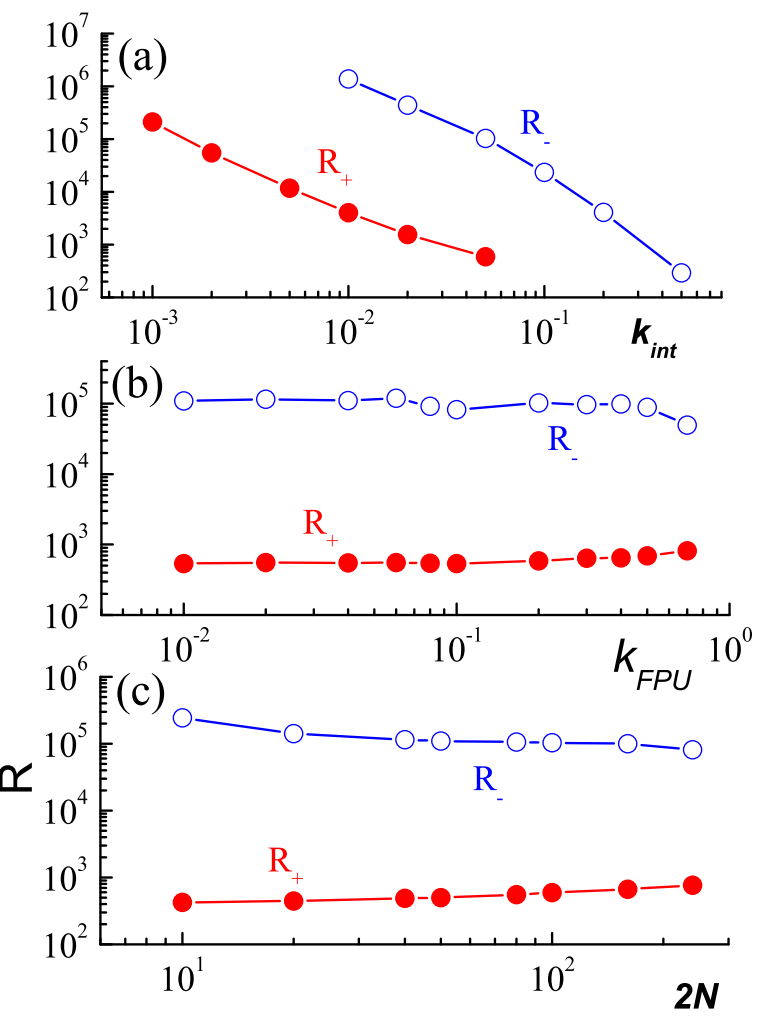

FIG. 6: (a) $R_{ \pm}$versus the coupling constant $k_{\text {int }}$. (b) $R_{ \pm}$ versus $k_{F P U}$. (c) $R_{ \pm}$versus the system size, $N$. For all three cases, $T_{0}=0.07$ and $|\Delta|=0.5 . \quad N=50$ for (a) and (b). $k_{\text {int }}=0.05$ for (b) and (c).

$R_{ \pm}$versus $k_{F P U}$ and Fig 6 (c) demonstrates the finite size effect. $R_{+}$increases slightly whereas $R_{-}$decreases slightly with $N$. This can be understood from the fact that with fixed $T_{+}$and $T_{-}$, when $\Delta>0$, the larger $N$ the smaller the $T_{i n t}^{L}$, thus the larger $R_{+}$; conversely, when $\Delta<0$, the larger $N$, the larger $T_{\text {int }}^{L}$ thus the smaller $R_{-}$.

The asymmetry of the ITR and the heat current in the model studied is due to the anharmonicity (nonlinearity) of the two lattices. In the low temperature limit, such as $T_{0}<0.01$, in which both the FPU and the FK models can be approximated by harmonic lattices, the asymmetry property will vanish because the phonon band of harmonic lattice is temperature independent. On the other hand, in the high temperature regime, in particular, when $T_{0}>V /(2 \pi)^{2}$ such that the on-site potential can be neglected, then the phonon band of the FK model becomes temperature independent. In this case, the asymmetry effect becomes minimal as we can see from Fig. 3 when $T_{0}>0.2$. However, the asymmetry will never disappear as long as the anharmonic term in the FPU model is still present.

In summary, we have studied the ITR between two dissimilar anharmonic lattices. It is found that the ITR is asymmetric, which is believed to be very general as the two anharmonic lattices used in this Letter are two representative ones widely studied in different fields of physics. The asymmetric property might be useful in heat control and management $[6]$. In particular, the very high ITR might find applications in building thermal insulator.

BL is supported in part by a FRG of NUS and the DSTA under Project Agreement POD0410553. LW is supported by DSTA POD0001821.

* Electronic address: phylibw@nus.edu.sg

[1] P. L. Kapitza, J. Phys. USSR 4, 181 (1941).

[2] E. T. Swartz and R. O. Pohl, Rev. Mod. Phys. 61, 605 (1989).

[3] G. Fang and C. Ward, Phys. Rev. E 59, 417, 441 (1999).

[4] A. Rosjorde et al. J. Colloid. Interface Sci. 232, 178 (2000); 240, 355 (2001); J. M. Simon et al. J. Phys. Chem. B 108, 7186 (2004).

[5] W. A. Little, Can. J. Phys. 37, 334 (1959).

[6] D. G. Cahill et al. J. App. Phys. 93, 793 (2003).

[7] D. K. Campbell, P. Rosenau, and G. Zaslavsky ed. "Focus Issue on 50'th anniversary of Fermi-Pasta-Ualam model", Chaos 15, no. 1, 015101-015121 (2005).

[8] O. M. Braun and Yu. S. Kivshar, "The FrenkelKontorova Model: Concepts, Methods, and Applications, Springer-Verlag, Berlin, 2003.

[9] H. Kaburaki and M. Machida, Phys. Lett. A181, 85 (1993); S. Lepri et al, Phys. Rev. Lett. 78, 1896 (1997); A. Fillipov, B. Hu, B. Li, and A. Zeltser, J. Phys. A 31. 7719 (1998), S. Lepri, Phys. Rev. E 58, 7165 (1998); K. Aoki and D. Kusnezov, Phys. Rev. Lett 86, 4029 (2001); A. Pereverzev, Phys. Rev. E, 68, 056124 (2003).

[10] B. Hu, B. Li, and H. Zhao, Phys. Rev. E 61, 3828 (2000).

[11] B. Hu, B. Li, and H. Zhao, Phys. Rev. E 57, 2992 (1998); A. V. Savin and O. V. Gendelman, Phys. Rev. E 67, 041205 (2003).

[12] F. Bonetto et al., in "Mathematical Physics 2000," A. Fokas et al. (eds) (Imperial College Press, London, 2000) (pp. 128-150); S. Lepri et al., Phys Rep. 377, 1 (2003).

[13] M. Terraneo, M. Peyrard, and G. Casati, Phys. Rev. Lett 88, 094302 (2002). B. Li, L. Wang, and G. Casati, Phys. 
Rev. Lett, 93, 184301 (2004). 